\title{
Effect of obesity and metabolic syndrome on plasma oxysterols and fatty acids in human
}

\author{
Marie Tremblay-Franco ${ }^{a}$, Chiara Zerbinati ${ }^{b}$, Antonio Pacelli ${ }^{b}$, Giuseppina Palmaccio ${ }^{b}$, Carla Lubrano ${ }^{c}$, \\ Simon Ducheix ${ }^{\mathrm{a}}$, Hervé Guillou ${ }^{\mathrm{a}}$, Luigi Iuliano ${ }^{\mathrm{b}, *}$ \\ a INRA, ToxAlim UMR1331 (Research Centre in Food Toxicology), Toulouse, France \\ ${ }^{\mathrm{b}}$ Department of Medico-Surgical Sciences and Biotechnologies, Sapienza University of Rome, Latina, Italy \\ ${ }^{\mathrm{c}}$ Department of Experimental Medicine, Sapienza University, Rome, Italy
}

\section{A R T I C L E I N F O}

\section{Article history:}

Received 2 January 2015

Received in revised form 19 March 2015

Accepted 23 March 2015

Available online 18 April 2015

\section{Keywords:}

Oxysterols

Fatty acids

Metabolic syndrome

Obesity

Diabetes

Lipid metabolism

\begin{abstract}
A B S T R A C T
Background: Obesity and the related entity metabolic syndrome are characterized by altered lipid metabolism and associated with increased morbidity risk for cardiovascular disease and cancer. Oxysterols belong to a large family of cholesterol-derived molecules known to play crucial role in many signaling pathways underlying several diseases. Little is known on the potential effect of obesity and metabolic syndrome on oxysterols in human.

Objectives: In this work, we questioned whether circulating oxysterols might be significantly altered in obese patients and in patients with metabolic syndrome. We also tested the potential correlation between circulating oxysterols and fatty acids.

Methods: 60 obese patients and 75 patients with metabolic syndrome were enrolled in the study along with 210 age- and sex-matched healthy subjects, used as control group. Plasma oxysterols were analyzed by isotope dilution GC/MS, and plasma fatty acids profiling was assessed by gas chromatography coupled with flame ionization detection.

Results: We found considerable differences in oxysterols profiling in the two disease groups that were gender-related. Compared to controls, males showed significant differences only in $4 \alpha$ - and $4 \beta$-hydroxycholesterol levels in obese and metabolic syndrome patients. In contrast, females showed consistent differences in 7-oxocholesterol, $4 \alpha$-hydroxycholesterol, 25-hydroxycholesterol and triol. Concerning fatty acids, we found minor differences in the levels of these variables in males of the three groups. Significant changes were observed in plasma fatty acid profile of female patients with obesity or metabolic syndrome. We found significant correlations between various oxysterols and fatty acids. In particular, $4 \beta$-hydroxycholesterol, which is reduced in obesity and metabolic syndrome, correlated with a number of saturated and mono-unsaturated fatty acids that are end-products of de novo lipogenesis. Conclusions: Our data provide the first evidence that obesity and metabolic syndrome are associated with major, gender-specific, changes in circulating oxysterols and fatty acids. These findings suggest a metabolic link between oxysterols and fatty acids, and that oxysterols may contribute to the epidemic diseases associated with obesity and metabolic syndrome in female.
\end{abstract}

(c) 2015 Elsevier Inc. All rights reserved.

\section{Introduction}

Obesity is a medical condition characterized by excessive accumulation of fat in the body and is better defined as a body mass index of 30 and above. It is a major epidemic and a public health concern worldwide [1]. Some obese patients may develop

* Corresponding author at: Sapienza University of Rome, Department of MedicoSurgical Sciences and Biotechnologies; Vascular Biology, Atherothrombosis \& Mass Spectrometry Lab, corso della Repubblica 79, 04100 Latina, Italy. Tel.: +39 0773 1757231; fax: +3906 62291089.

E-mail address: luigi.iuliano@uniroma1.it (L. Iuliano). important complications, including the metabolic syndrome, that favor premature death [2]. Metabolic syndrome is the most studied complication in obesity and is diagnosed by the co-occurrence of three out of five of the following conditions: abdominal (central) obesity, high blood pressure, high fasting plasma glucose, high serum triglycerides, and low high-density cholesterol (HDL) levels [3]. Additional complications such as liver disorders (non alcoholic fatty liver diseases) [4], inflammatory bowel diseases [5], reproductive disorders including infertility [6], autoimmune diseases [7] and many cancers [8] may also develop in obesity. Interestingly, preclinical and clinical studies have provided 
evidence for the part played by lipids in the etiology of diseases associated with obesity.

Oxysterols are a complex family of oxidized species of cholesterol [9]. They are powerful bioactive lipids able to influence an array of biological processes [10] and may be involved in the pathologies associated with obesity. There are several mechanisms by which oxysterols influence cell signaling and physiology. Oxysterols are potent regulators of cholesterogenic pathways [11] and they act as ligands for receptors such as the Liver $X$ Receptors (LXRs) [12], which are nuclear receptors that act as oxysterol sensors and regulate gene expression [13]. They are highly expressed in the liver where they regulate the expression of genes involved in cholesterol and fatty acid metabolism [14]. More recently, it has also been shown that 27-hydroxycholesterol act as selective estrogen receptor modulators (SERM) [15] and influence the cardiovascular system, bone biology and cancer [16].

There are little data on the relationship between oxysterol and metabolic diseases in human. In this work we investigated whether obesity and metabolic syndrome might have a significant impact on serum oxysterol profile. Because cholesterol metabolism shows important gender specificity and oxysterols act as SERMs we questioned this both in males and in females. In addition, various independent works provided evidence that not only fatty acid metabolism is regulated by oxysterol receptors (LXRs) $[14,17]$ but also that fatty acids influence LXR activity $[18,19]$. Therefore, we tested whether changes in oxysterol concentrations may be correlated to changes in circulating fatty acids.

\section{Methods}

\subsection{Study population and design}

To investigate the characteristics of plasma FA and oxysterols pattern in patients with obesity and metabolic syndrome we consecutively recruited 142 subjects at Sapienza University outpatient centers for Obesity and Vascular Medicine (obesity patients, $n=67$; MS, $n=75$ ) between January 2009 and July 2011. Inclusion criteria for obese patients were based on BMI > 30 in the absence of dyslipidemia, diabetes or hypertension. Diagnosis of metabolic syndrome was based on the ATPIII criteria [3]. Exclusion criteria included, previous angina, myocardial infarction, TIA or stroke, familial dyslipidemias, thyroid disease, kidney disease, systemic inflammatory diseases or cancer.

Healthy controls $(n=210)$, matched for age and sex, were recruited in the same geographical area, after carefully reviewing their medical history, among blood donors referring to the AVIS center of Latina. Healthy subject definition was based on the absence of any known clinical or anamnestic disease, and the absence of cardiovascular risk factors apart from smoking.

The study procedure was developed according to the guidelines of the Ethic Committee, which approved the protocol, and the Helsinki Declaration of 1975 . All subjects signed written informed consent.

\subsection{Plasma biochemistry}

Serum glucose, bilirubin, uric acid, alanine transaminase, cholesterol, triglycerides, HDL-and cholesterol were determined by standard automated techniques as previously described [20]. Laboratory analyses were done in a blinded fashion. The clinical picture of the patients referred to in this study is described in Table 1.

\subsection{Lipid biochemistry}

To measure plasma FAs, peripheral venous blood was obtained after overnight fasting and was centrifuged at $4{ }^{\circ} \mathrm{C}$. Plasma was then removed and stored at $-80^{\circ} \mathrm{C}$ until assay. Before analysis FAs were processed for direct transesterification with acetyl chloride according to a previously published method [21]. Analyses were performed on an Agilent 7820A Plus Gas Chromatograph (Agilent Technologies; Palo Alto, CA) equipped with a G4513A automatic liquid sampler and a flame ionization detector. Separation was carried out on a $100 \mathrm{~m}$ capillary column (Supelco, SP-2560 $100 \mathrm{~m} \times 0.25 \mathrm{~mm}$ ID, $0.20 \mu \mathrm{m}$ thickness) (Sigma Aldrich, Milan, Italy). Identification, precision, and accuracy were evaluated using mixtures of authentic methylated FA standards, and a control plasma pool as previously described [22,23]. The list of FAs species that were analysed is reported in Supplementary Table 1.

Oxysterols were determined by GC-MS using deuteriumlabelled internal standards, as previously described [24]. Prior to analysis the isolated oxysterol fraction was submitted to base hydrolysis, hence the concentrations measured are for total oxysterols comprising the sum of esterified and non-esterified molecules. 5 $\alpha-\mathrm{OH}, 6$-oxocholesterol, d6-5 $\alpha-\mathrm{OH}, 6-\mathrm{oxocholesterol}$ cholestane, triol and d6-triol were synthesized and kindly donated by Dr. Marc Poirot (Institut Claudius Régaud, Toulouse, France). $4 \alpha$ - and $4 \beta \mathrm{OH}$-Cholesterol were kindly donated by Dr. G. Lizard (Faculté des Sciences Gabriel, Dijon, France). All other oxysterols and deuterated oxysterols were from Avanti Polar Lipids. The list of oxysterols measured here is reported in Supplementary Table 2.

\subsection{Statistical analysis}

Kruskal-Wallis test was used to compare oxysterol and fatty acid concentrations between the three health status groups (healthy, obesity, metabolic syndrome). This is a non-parametric univariate test that treats each variable independently. Multiple testing corrections (adjusted $p$-values), the false discovery rate, were applied to avoid false positive [25].

A heatmap was used to represent relationships between fatty acids and oxysterols. This is a graphical representation of Kendall correlation coefficients using darker colors to indicate high correlations, and brighter colors to indicate low correlations. Statistical analysis was performed using $\mathrm{R}$ software (http://www.r-project. $\operatorname{org} /$ ).

\section{Results}

\subsection{Clinical characteristics of the study population}

Based on the ATPIII criteria, participants were classified as healthy controls, metabolic syndrome or obesity patients. We used these groups to investigate the respective influence of obesity and metabolic syndrome on plasma oxysterols and fatty acids. Demographic and clinical characteristics of the study population are shown in Table 1. Oxysterol profile showed significant differences in the three groups (Supplementary Table 3). However, analysis performed independently in patients from each gender revealed that these changes were markedly gender-dependent.

\subsection{Influence of obesity and metabolic syndrome on plasma oxysterol concentration in males}

Interestingly, we found that obesity alone has little impact on plasma oxysterols concentration when compared to healthy controls (Table 2). Only $4 \beta$-hydroxycholesterol and $4 \alpha$-hydroxycholesterol were significantly different in males. Both $4 \beta$-hydroxycholesterol and $4 \alpha$-hydroxycholesterol were lower in obese patients compared to healthy subjects. In patients with metabolic syndrome the level of $4 \beta$-hydroxycholesterol was lower and the level of and $4 \alpha$-hydroxycholesterol was higher compared to healthy 
Table 1

Demographic and clinical characteristics of the study population.

\begin{tabular}{|c|c|c|c|c|c|c|c|c|}
\hline & \multirow{2}{*}{$\begin{array}{l}\mathrm{HC} \\
(n=210)\end{array}$} & \multirow{2}{*}{$\begin{array}{l}\text { OB } \\
(n=67)\end{array}$} & \multirow{2}{*}{$\begin{array}{l}\text { MS } \\
(n=75)\end{array}$} & \multicolumn{2}{|c|}{ ANOVA } & \multirow[t]{2}{*}{$\mathrm{HC}$ vs. OB } & \multirow[t]{2}{*}{ HC vs. MS } & \multirow[t]{2}{*}{ MS vs. OB } \\
\hline & & & & $F$ & $p$ & & & \\
\hline Age (years) & $41.7 \pm 11.4$ & $40.5 \pm 12.2$ & $45.9 \pm 10.1$ & 4.8 & 0.008 & n.s. & 0.02 & 0.016 \\
\hline BMI $\left(\mathrm{kg} / \mathrm{m}^{2}\right)$ & $25.3 \pm 2.4$ & $34.9 \pm 4.7$ & $35.1 \pm 6.0$ & 251.8 & $<0.0001$ & $<0.0001$ & $<0.0001$ & n.s. \\
\hline Male sex (\%) & $136(64)$ & $28(41)$ & $41(54)$ & & & & & \\
\hline $\mathrm{SBP}(\mathrm{mmHg})$ & $120 \pm 12$ & $120 \pm 12$ & $135 \pm 14$ & 38.7 & $<0.0001$ & n.s. & $<0.0001$ & $<0.0001$ \\
\hline DBP (mmHg) & $78 \pm 8$ & $78 \pm 9$ & $87 \pm 9$ & 35.5 & $<0.0001$ & n.s. & $<0.0001$ & $<0.0001$ \\
\hline $\mathrm{TC}(\mathrm{mg} / \mathrm{dL})^{*}$ & $198.89 \pm 37.53$ & $184.97 \pm 37.79$ & $210.27 \pm 42.97$ & 7.5 & 0.001 & 0.01 & 0,03 & $<0.0001$ \\
\hline HDL-c (mg/dL) & $57.6 \pm 13.5$ & $49.9 \pm 10.4$ & $46.8 \pm 11.3$ & 24.8 & $<0.0001$ & $<0.0001$ & $<0.0001$ & n.s. \\
\hline $\mathrm{TG}(\mathrm{mg} / \mathrm{dL})^{*}$ & $86.09 \pm 36.87$ & $93.09 \pm 38.91$ & $143.43 \pm 69.48$ & 43.6 & $<0.0001$ & n.s. & $<0.0001$ & $<0.0001$ \\
\hline
\end{tabular}

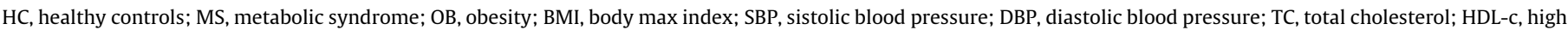
density lipoprotein-cholesterol; TG, triglycerides; n.s., not significant. Values are expressed as mean \pm SD; LSD was used for post hoc comparison between groups.

To convert TC and HDL-c values in mmol/L divide by 38.67; to convert TG values in mmol/L divide by 88.57 .

Table 2

Concentration of serum cholesterol and oxysterols measured in males by health status.

\begin{tabular}{|c|c|c|c|c|c|c|c|}
\hline & $\mathrm{HC}$ & $\mathrm{OB}$ & MS & $p^{1}$ & $\mathrm{HC} / \mathrm{OB}$ & $\begin{array}{l}\mathrm{HCl} \\
\mathrm{MS}\end{array}$ & $\begin{array}{l}\mathrm{OB} / \\
\mathrm{MS}\end{array}$ \\
\hline $\mathrm{TC}(\mathrm{mg} / \mathrm{dL})$ & $199.23 \pm 39.02$ & $201.95 \pm 29.41$ & $209.57 \pm 41.78$ & 0.1978 & n.s. & n.s. & n.s. \\
\hline $4 \beta \mathrm{OH}-\mathrm{CH}$ & $17.77 \pm 5.22$ & $13.33 \pm 2.80$ & $16.16 \pm 5.49$ & 0.0019 & 0.0083 & 0.0186 & n.s. \\
\hline $4 \beta \mathrm{OH}-\mathrm{CH} / \mathrm{TC}$ & $9.06 \pm 2.54$ & $6.71 \pm 1.65$ & $7.84 \pm 2.52$ & 0.0001 & 0.0004 & 0.0004 & n.s. \\
\hline $5 \propto \mathrm{OH}, 6$ охо-CH & $1.30 \pm 0.32$ & $1.49 \pm 0.55$ & $1.43 \pm 0.44$ & 0.1330 & n.s. & n.s. & n.s. \\
\hline $5 \alpha \mathrm{OH}, 60 \times 0-\mathrm{CH} / \mathrm{TC}$ & $0.67 \pm 0.21$ & $0.75 \pm 0.30$ & $0.70 \pm 0.23$ & 0.8472 & n.s. & n.s. & n.s. \\
\hline 5,6 $\alpha$-ероху & $11.13 \pm 9.55$ & $9.55 \pm 3.10$ & $10.45 \pm 3.39$ & 0.3347 & n.s. & n.s. & n.s. \\
\hline 5,6 $\alpha$-ероху/TC & $5.69 \pm 4.29$ & $4.78 \pm 1.65$ & $5.1 \pm 1.75$ & 0.8472 & n.s. & n.s. & n.s. \\
\hline 5,6ß-ероху & $20.62 \pm 10.78$ & $19.00 \pm 6.59$ & $21.28 \pm 8.15$ & 0.3347 & n.s. & n.s. & n.s. \\
\hline 5,6ß-ероху/TC & $10.65 \pm 5.44$ & $9.43 \pm 3.04$ & $10.42 \pm 4.51$ & 0.8472 & n.s. & n.s. & n.s. \\
\hline $27 \mathrm{OH}-\mathrm{CH}$ & $59.66 \pm 15.12$ & $58.28 \pm 12.67$ & $63.06 \pm 18.32$ & 0.3347 & n.s. & n.s. & n.s. \\
\hline $27 \mathrm{OH}-\mathrm{CH} / \mathrm{TC}$ & $30.77 \pm 8.68$ & $19.12 \pm 6.66$ & $30.77 \pm 8.74$ & 0.8472 & n.s. & n.s. & n.s. \\
\hline $7 \alpha \mathrm{OH}-\mathrm{CH}$ & $36.03 \pm 23.96$ & $40.79 \pm 27.35$ & $43.96 \pm 24.79$ & 0.1330 & n.s. & n.s. & n.s. \\
\hline $7 \propto \mathrm{OH}-\mathrm{CH} / \mathrm{TC}$ & $17.10 \pm 10.97$ & $20.24 \pm 13.37$ & $21.24 \pm 12.09$ & 0.6313 & n.s. & n.s. & n.s. \\
\hline Triol & $2.74 \pm 0.80$ & $2.54 \pm 0.88$ & $2.99 \pm 1.16$ & 0.1330 & n.s. & n.s. & n.s. \\
\hline Triol/TC & $1.41 \pm 0.5$ & $1.29 \pm 0.55$ & $1.47 \pm 0.58$ & 0.6313 & n.s. & n.s. & n.s. \\
\hline $25 \mathrm{OH}-\mathrm{CH}$ & $6.55 \pm 1.55$ & $6.69 \pm 1.19$ & $6.91 \pm 1.55$ & 0.3347 & n.s. & n.s. & n.s. \\
\hline $25 \mathrm{OH}-\mathrm{CH} / \mathrm{TC}$ & $3.32 \pm 0.63$ & $3.33 \pm 0.51$ & $3.34 \pm 0.66$ & 0.9324 & n.s. & n.s. & n.s. \\
\hline $7 \beta \mathrm{OH}-\mathrm{CH}$ & $13.03 \pm 10.57$ & $11.98 \pm 2.58$ & $13.43 \pm 4.61$ & 0.1606 & n.s. & n.s. & n.s. \\
\hline $7 \beta \mathrm{OH}-\mathrm{CH} / \mathrm{TC}$ & $6.63 \pm 4.87$ & $5.96 \pm 1.16$ & $6.53 \pm 2.12$ & 0.8472 & n.s. & n.s. & n.s. \\
\hline $4 \alpha \mathrm{OH}-\mathrm{CH}$ & $5.38 \pm 2.07$ & $4.81 \pm 1.09$ & $5.80 \pm 1.26$ & 0.0019 & n.s. & 0.0023 & 0.0027 \\
\hline $4 \alpha \mathrm{OH}-\mathrm{CH} / \mathrm{TC}$ & $2.74 \pm 1.03$ & $2.40 \pm 0.49$ & $2.82 \pm 0.63$ & 0.0866 & n.s. & n.s. & n.s. \\
\hline $24 \mathrm{OH}-\mathrm{CH}$ & $45.51 \pm 11.69$ & $46.74 \pm 11.74$ & $46.78 \pm 12.12$ & 0.8184 & n.s. & n.s. & n.s. \\
\hline $24 \mathrm{OH}-\mathrm{CH} / \mathrm{TC}$ & $23.41 \pm 6.54$ & $23.13 \pm 4.85$ & $22.76 \pm 5.92$ & 0.8472 & n.s. & n.s. & n.s. \\
\hline 7oxo-CH & $12.79 \pm 10.76$ & $12.51 \pm 3.06$ & $13.03 \pm 3.46$ & 0.1195 & n.s. & n.s. & n.s. \\
\hline 7oxo-CH/TC & $6.56 \pm 5.02$ & $6.2 \pm 1.35$ & $6.34 \pm 1.59$ & 0.8472 & n.s. & n.s. & n.s. \\
\hline
\end{tabular}

HC, healthy controls; MS, metabolic syndrome; OB, obesity; TC, total cholesterol; oxysterols (ng/mL); oxysterols/TC (ng/mg); n.s., not significant.

${ }^{1}$ False Discovery Rate adjusted $p$-value of Kruskal-Wallis test.

controls. Consistent with a minor effect of metabolic syndrome on oxysterols, we show that only $4 \alpha$-hydroxycholesterol was significantly different between obesity and metabolic syndrome patients.

\subsection{Influence of obesity and metabolic syndrome on the serum oxysterol concentration in females}

A completely different picture of oxysterols profile was observed in females. Obese patients group showed lower levels of 5,6 $\alpha$-epoxycholesterol, $4 \beta$-hydroxycholesterol and 24-hydroxycholesterol, compared to healthy subjects (Table 3). Metabolic syndrome group showed lower levels of $4 \beta$-hydroxysterol, $5,6 \alpha$-epoxycholesterol, compared to healthy controls. Differently from males, females carrying the features of metabolic syndrome showed significantly higher concentrations of six oxysterols when compared to healthy patients. Importantly the ratios between oxysterols and cholesterol were also significantly different which implies that changes in oxysterols were not just reflection of changes in cholesterol levels. These oxysterols included $7 \alpha$-hydroxycholesterol, 7 $\beta$-hydroxycholesterol, 7-oxocholesterol, triol, $4 \alpha$-hydroxycholesterol and 25-hydroxycholesterol. Amongst them, triol and 7 $\beta$-hydroxycholesterol were significantly higher in patients with metabolic syndrome when compared to obese patients. However, the ratios between these oxysterol and cholesterol were found not to be significantly different.

\subsection{Relationship between plasma fatty acids and oxysterols}

The fatty acid profiles from healthy controls, obese patients and patients with metabolic syndrome are reported in Supplementary Tables 4 and 5 . In the male population, we found little changes in fatty acids whatever the clinical picture of the patients was considered. Adjusted $p$-values revealed that none of the fatty acids was significantly related to the disease state (Supplementary Table 4). In contrast, in females we identified significant changes in the fatty acid profile of patients with obesity and metabolic syndrome (Supplementary Table 5). In particular, compared to healthy controls, obese patients showed higher levels of C18:0, C24:0, C18:1n-7, C24:1n-9, C20:4n-6, C22:4n-6 and C22:5n-6, and lower levels of $\mathrm{C} 14: 1, \mathrm{C} 18: 2 \mathrm{n}-6$ and $\mathrm{C} 20: 3 \mathrm{n}-3$. The same picture was observed in female patients with metabolic syndrome compared 
Table 3

Concentration of serum cholesterol and oxysterols measured in females by health status.

\begin{tabular}{|c|c|c|c|c|c|c|c|}
\hline & $\mathrm{HC}$ & $\mathrm{OB}$ & MS & $p^{1}$ & $\mathrm{HC} / \mathrm{OB}$ & $\mathrm{HC} / \mathrm{MS}$ & $\mathrm{OB} / \mathrm{MS}$ \\
\hline $\mathrm{TC}(\mathrm{mg} / \mathrm{dL})$ & $197.77 \pm 37.46$ & $178.21 \pm 41.85$ & $191.66 \pm 38.69$ & 0.1322 & n.s. & n.s. & n.s. \\
\hline $4 \beta \mathrm{OH}-\mathrm{CH}$ & $21.99 \pm 7.25$ & $15.90 \pm 5.77$ & $16.20 \pm 7.23$ & $<0.0001$ & 0.0008 & $<0.0001$ & n.s. \\
\hline $4 \beta \mathrm{OH}-\mathrm{CH} / \mathrm{TC}$ & $11.28 \pm 3.47$ & $9.34 \pm 4.32$ & $8.48 \pm 3.21$ & $<0.0001$ & 0.0061 & $<0.0001$ & n.s. \\
\hline $5 \alpha \mathrm{OH}, 6$ охо- $\mathrm{CH}$ & $1.05 \pm 0.34$ & $0.96 \pm 0.24$ & $1.04 \pm 0.28$ & 0.6250 & n.s. & n.s. & n.s. \\
\hline $5 \propto \mathrm{OH}, 6$ oxo-CH/TC & $0.54 \pm 0.15$ & $0.55 \pm 0.12$ & $0.55 \pm 0.14$ & 0.7325 & n.s. & n.s. & n.s. \\
\hline 5,6a-epoxy & $7.57 \pm 4.43$ & $5.84 \pm 3.23$ & $5.25 \pm 2.35$ & 0.0003 & 0.017 & $<0.0001$ & n.s. \\
\hline 5,6 $\alpha$-epoxy/TC & $3.87 \pm 2.11$ & $3.43 \pm 1.92$ & $2.78 \pm 1.09$ & 0.0033 & n.s. & 0.0010 & n.s. \\
\hline 5,6ß-ероху & $12.54 \pm 5.37$ & $10.93 \pm 3.72$ & $10.60 \pm 3.26$ & 0.1216 & n.s. & n.s. & n.s. \\
\hline 5,6ß-ероху/TC & $6.42 \pm 2.50$ & $6.46 \pm 2.56$ & $5.66 \pm 1.85$ & 0.2972 & n.s. & n.s. & n.s. \\
\hline $27 \mathrm{OH}-\mathrm{CH}$ & $46.34 \pm 11.45$ & $42.71 \pm 8.80$ & $41.15 \pm 10.78$ & 0.0893 & n.s. & n.s. & n.s. \\
\hline $270 \mathrm{H}-\mathrm{CH} / \mathrm{TC}$ & $24.08 \pm 6.80$ & $24.90 \pm 6.09$ & $21.88 \pm 5.73$ & 0.0946 & n.s. & n.s. & n.s. \\
\hline $7 \alpha \mathrm{OH}-\mathrm{CH}$ & $23.56 \pm 13.66$ & $23.03 \pm 12.89$ & $32.79 \pm 18.69$ & 0.0176 & n.s. & 0.014 & n.s. \\
\hline $7 \alpha \mathrm{OH}-\mathrm{CH} / \mathrm{TC}$ & $11.71 \pm 5.57$ & $14.47 \pm 13.06$ & $17.18 \pm 10.15$ & 0.0054 & n.s. & 0.0029 & n.s. \\
\hline Triol & $2.90 \pm 1.09$ & $2.88 \pm 0.88$ & $3.47 \pm 0.76$ & 0.0011 & n.s. & 0.0005 & 0.0097 \\
\hline Triol/TC & $1.52 \pm 0.66$ & $1.70 \pm 0.70$ & $1.88 \pm 0.56$ & 0.0008 & n.s. & 0.0002 & n.s. \\
\hline $25 \mathrm{OH}-\mathrm{CH}$ & $6.74 \pm 1.24$ & $6.82 \pm 1.34$ & $7.46 \pm 1.32$ & 0.0176 & n.s. & 0.013 & n.s. \\
\hline $250 \mathrm{H}-\mathrm{CH} / \mathrm{TC}$ & $3.47 \pm 0.61$ & $3.97 \pm 0.98$ & $3.97 \pm 0.69$ & 0.0006 & 0.0168 & 0.0002 & n.s. \\
\hline $4 \alpha \mathrm{OH}-\mathrm{CH}$ & $4.90 \pm 1.04$ & $5.46 \pm 2.30$ & $5.53 \pm 1.41$ & 0.0448 & n.s. & 0.033 & n.s. \\
\hline $4 \propto \mathrm{OH}-\mathrm{CH} / \mathrm{TC}$ & $2.51 \pm 0.43$ & $3.18 \pm 1.44$ & $2.95 \pm 0.83$ & 0.0019 & 0.0106 & 0.0021 & n.s. \\
\hline $7 \beta \mathrm{OH}-\mathrm{CH}$ & $13.18 \pm 3.61$ & $13.11 \pm 2.23$ & $15.93 \pm 3.85$ & 0.0003 & n.s. & $<0.0001$ & 0.0042 \\
\hline $7 \beta \mathrm{OH}-\mathrm{CH} / \mathrm{TC}$ & $6.79 \pm 1.51$ & $7.82 \pm 2.62$ & $8.48 \pm 2.24$ & $<0.0001$ & n.s. & $<0.0001$ & n.s. \\
\hline $24 \mathrm{OH}-\mathrm{CH}$ & $47.58 \pm 11.16$ & $39.23 \pm 8.56$ & $45.86 \pm 14.89$ & 0.0197 & 0.01 & n.s. & n.s. \\
\hline $24 \mathrm{OH}-\mathrm{CH} / \mathrm{TC}$ & $24.71 \pm 6.90$ & $22.59 \pm 4.84$ & $23.99 \pm 5.70$ & 0.7395 & n.s. & n.s. & n.s. \\
\hline 7oxo-CH & $10.56 \pm 3.25$ & $10.26 \pm 2.46$ & $12.14 \pm 3.58$ & 0.0141 & n.s. & 0.012 & n.s. \\
\hline 7oxo-CH/TC & $5.39 \pm 1.46$ & $6.32 \pm 3.50$ & $6.51 \pm 2.20$ & 0.0022 & n.s. & 0.0005 & n.s. \\
\hline
\end{tabular}

HC, healthy controls; MS, metabolic syndrome; OB, obesity; TC, total cholesterol; oxysterols (ng/mL); oxysterols/TC (ng/mg); n.s., not significant.

1 False Discovery Rate adjusted $p$-value of Kruskal-Wallis test.

to healthy controls. Obesity resulted in higher levels of C20:4n-6 to metabolic syndrome.

Next, we questioned whether individual measures of oxysterols might correlate with some fatty acids. We identified significant correlations between various oxysterols and fatty acids (Fig. 1). Interestingly, $4 \beta$-hydroxysterol that is reduced in obesity and in metabolic syndrome markedly correlated with a number of fatty acids that are end-products of de novo lipogenesis (C14:0, C16:0, C16:1n-7 and $(18: 1 n-9)$ both in males and in females (Supplementary Figs. 1 and 2). While some of these fatty acids are part of the diet, ratios between products and substrates are used as indicators of Stearoyl-CoA Desaturase activities. We calculated these ratios and found that consistent with the gender-specific effect on oxysterols there is also a marked gender-specific effect of mono-unsaturated to saturated ratios (Supplementary Fig. 3).

\section{Discussion}

Growing evidence indicates that obesity epidemic is associated with increased prevalence of a number diseases - including liver, reproductive system and autoimmune disorders as well as cancer [4-8]. Many lipid molecular species have been implicated in the etiology of obesity associated diseases. The best known examples are fatty acids that may influence lipotoxicity in insulin-sensitive tissues and play a part in the development of type II diabetes [26-28].

Little is known about the possible role of oxysterols, a family of highly bioactive lipids $[23,29,30]$, in obesity and obesity-related diseases. Experimental models provided evidence that oxysterols control fatty acid homeostasis by acting as ligands for transcription factors, i.e. LXRs, that regulate whole body lipid homeostasis $[14,17]$. In the present study, we measured circulating oxysterols and fatty acids in patients with obesity and metabolic syndrome, healthy subjects serving as control, looking at differences and potential correlation between the two lipid systems.
Compared to healthy controls, we found gender-related distribution of circulating oxysterols and fatty acids in patients with obesity and metabolic syndrome. Concerning oxysterols levels in male patients, we found significant changes in $4 \alpha$ - and $4 \beta$-hydroxycholesterol levels only. Similar findings were observed in females but, in addition, significant changes were observed in several oxysterols of both enzymatic and non-enzymatic origin. In particular, higher levels of $7 \beta$-hydroxycholesterol and triol were found in female patients with metabolic syndrome compared to healthy controls or obese patients. These observations are consistent with the well-known sexual dimorphism in lipid metabolism [31]. To our knowledge, only 7-oxysterols were reported as possible markers of atherogenic dyslipidemia in adolescence [32]. Whether the changes in oxysterols reported in males and in females might be promoting diseases associated with obesity or with metabolic it remains to be determined. However, the hypothesis that oxysterols may be involved in sex-specific disease progression is worthy of further investigation.

Similar to the oxysterol profiles, we found that the fatty acid pattern was profoundly altered in female but not in male patients with obesity and metabolic syndrome. Several components of the saturated, monounsaturated and n- 6 polyunsaturated FA were found altered in the two disease groups compared to healthy controls. The n-3 FA class did not show relevant changes apart from C20:3n-3 that was reduced in both obesity and metabolic syndrome. Similarly to the Atherosclerosis Risk in Community (ARIC) study [33], we found reduced levels of linoleic acid (C18:2n-6) pointing to a potentially imbalanced dietary intake or to a defect in the metabolism of essential fatty acids in female patients with obesity and metabolic syndrome.

There has been a lot if interest in the role of palmitoleic acid (C16:1n-7) as an endogenous lipid mediator [34]. Our data show significantly higher levels of palmitoleic acid in female patients with metabolic syndrome. These findings are in agreement with the ARIC Study [33] but put into question the beneficial effects of this potential lipokine in female. Since C16:1n-7 shows such a 

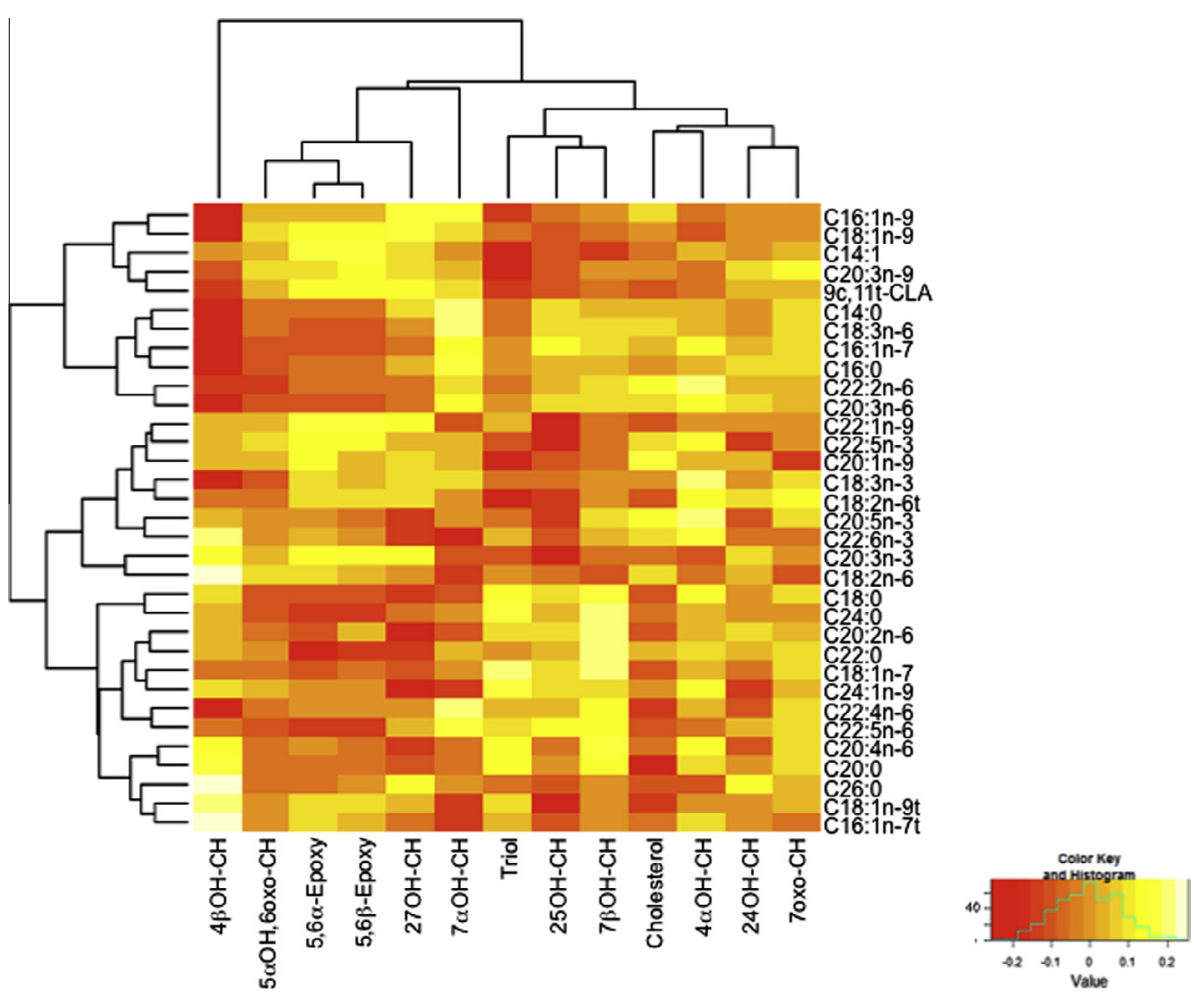

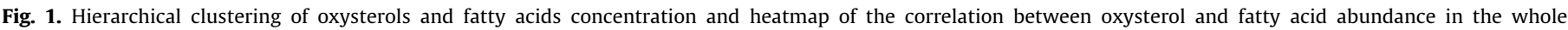

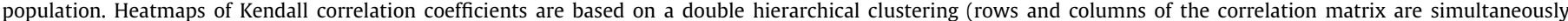

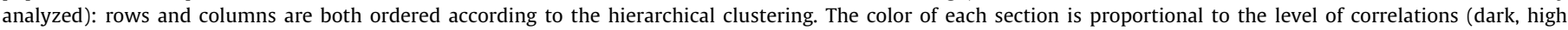
correlation; bright, low correlation).

specific profile in females, it would be important to consider sexually dimorphic regulation of the key enzymes involved in palmitoleate metabolism [35]. To the best of our knowledge, little is known about the gender-specific activity of $\Delta 9$-desaturase (Stearoyl-CoA Desaturases) and Elongase (Elovl6) in lipogenic human tissues such as the liver and the white adipose tissues. Therefore, we calculated ratios between the abundance of monounsaturated fatty acids of the n-7 and n-9 series and the abundance of their respective saturated precursor. We found a significant effect of obesity and metabolic syndrome on both ratios but only in females, which is consistent with a gender-specific regulation of lipogenesis. In addition, we found that both ratios behave oppositely. While the $n-9$ ratio decreases, the $n-7$ ratio increases. The $\mathrm{n}-7$ ratio is a better indicator of $\Delta 9$-desaturas activity because in the $n-9$ series, a lot of circulating C18:1n-9 may be provided through the diet. The increase in n-7 ratio in obese and in patients with metabolic syndrome may be indicative of increased lipogenesis and, possibly, non alcoholic fatty liver disease, which is highly prevalent in patients with obesity and metabolic syndrome [36].

Finally, we identified statistically significant relationships between circulating fatty acids and oxysterols. In human, this observation is consistent with the evidence provided by pre-clinical studies showing that the oxysterol receptor LXR drives de novo lipogenic genes [14,17] and dietary fatty acids may impact on LXR activity [18,19]. Interestingly, the most striking correlation observed were those linking $4 \beta$-hydroxycholesterol to saturated and mono-unsaturated fatty acids, both in males and females. $4 \beta$-hydroxycholesterol has a unusually long half-life in human plasma due to slow $7 \alpha$-hydroxylation required for its clearance [37] and it is formed by the drug-metabolizing enzyme cytochrome P450 3A4 [38]. It is therefore possible that lipogenic genes and $4 \beta$-hydroxycholesterol degrading enzymes are co-regulated in humans. Another possibility is that a regulator of lipogenesis or fatty acid(s) produced by de novo lipogenesis may inhibit Cyp3A4 expression and/or activity.

Altogether, we provide the first report showing the complete picture of oxysterol and FA profile in patients with obesity and metabolic syndrome. Data reported here support gender-specific oxysterol profiles that should be taken into account in the analysis and treatment of human diseases associated with obesity and metabolic syndrome.

\section{Acknowledgements}

The laboratory involved in this work are members of the European Network for Oxysterol Research (ENOR) and wish to thank the ENOR members for their input and for fruitful discussions. HG is supported by a grant from the ANR - France Crisalis (2012-2015). This work was supported in part by a grant from Sapienza University of Rome - Italy (Grant Ateneo 2013 \# C26A13YNTT, to LI).

\section{Appendix A. Supplementary data}

Supplementary data associated with this article can be found, in the online version, at http://dx.doi.org/10.1016/j.steroids.2015.03. 019 .

\section{References}

[1] Sellayah D, Cagampang FR, Cox RD. On the evolutionary origins of obesity: a new hypothesis. Endocrinology 2014:155:1573-88.

[2] Murdolo G, Piroddi M, Luchetti F, et al. Oxidative stress and lipid peroxidation by-products at the crossroad between adipose organ dysregulation and obesity-linked insulin resistance. Biochimie 2013:95:585-94.

[3] Third report of the national cholesterol education program (NCEP) expert panel on detection, evaluation, and treatment of high blood cholesterol in 
adults (adult treatment panel III) final report. Circulation 2002;106:31433421.

[4] Chamorro-Garcia R, Blumberg B. Transgenerational effects of obesogens and the obesity epidemic. Curr Opin Pharmacol 2014;19C:153-8.

[5] Kredel LI, Siegmund B. Adipose-tissue and intestinal inflammation - visceral obesity and creeping fat. Front Immunol 2014;5:1-11.

[6] Crujeiras AB, Casanueva FF. Obesity and the reproductive system disorders: epigenetics as a potential bridge. Hum Reprod Update; 2014 [Epub ahead of print].

[7] Versini M, Jeandel PY, Rosenthal E, et al. Obesity in autoimmune diseases: not a passive bystander. Autoimmun Rev 2014;13:981-1000.

[8] Goodwin PJ, Stambolic V. Impact of the obesity epidemic on cancer. Annu Rev Med; 2014 [Epub ahead of print].

[9] Russell DW. Oxysterol biosynthetic enzymes. Biochim Biophys Acta 2000;1529:126-35.

[10] Schroepfer GJJ. Oxysterols: modulators of cholesterol metabolism and other processes. Physiol Rev 2000;80:361-554.

[11] Brown AJ, Jessup W. Oxysterols: sources, cellular storage and metabolism, and new insights into their roles in cholesterol homeostasis. Mol Aspects Med 2009;30:111-22.

[12] Janowski BA, Willy PJ, Devi TR, et al. An oxysterol signalling pathway mediated by the nuclear receptor LXR alpha. Nature 1996;383:728-31.

[13] Janowski BA, Grogan MJ, Jones SA, et al. Structural requirements of ligands for the oxysterol liver X receptors LXRalpha and LXRbeta. Proc Natl Acad Sci USA 1999;96:266-71.

[14] Repa JJ, Liang G, Ou J, et al. Regulation of mouse sterol regulatory elementbinding protein-1c gene (SREBP-1c) by oxysterol receptors LXRalpha and LXRbeta. Genes Dev 2000;14:2819-30.

[15] Umetani M, Domoto H, Gormley AK, et al. 27-Hydroxycholesterol is an endogenous SERM that inhibits the cardiovascular effects of estrogen. Nat Med 2007; 13:1185-92.

[16] Nelson ER, Wardell SE, McDonnell DP. The molecular mechanisms underlying the pharmacological actions of estrogens, SERMs and oxysterols: implications for the treatment and prevention of osteoporosis. Bone 2013;53:42-50.

[17] Beaven SW, Matveyenko A, Wroblewski K, et al. Reciprocal regulation of hepatic and adipose lipogenesis by liver $\mathrm{X}$ receptors in obesity and insulin resistance. Cell Metab 2013;18:106-17.

[18] Ducheix S, Montagner A, Polizzi A, et al. Essential fatty acids deficiency promotes lipogenic gene expression and hepatic steatosis through the liver $\mathrm{X}$ receptor. J Hepatol 2013;58:984-92.

[19] Ou J, Tu H, Shan B, et al. Unsaturated fatty acids inhibit transcription of the sterol regulatory element-binding protein-1c (SREBP-1c) gene by antagonizing ligand-dependent activation of the LXR. Proc Natl Acad Sci USA 2001:98:6027-32.

[20] Ginanni Corradini S, Zerbinati C, Maldarelli F, et al. Plasma fatty acid lipidome is associated with cirrhosis prognosis and graft damage in liver transplantation. Am J Clin Nutr 2014;100:600-8.
[21] Masood A, Stark KD, Salem NJ. A simplified and efficient method for the analysis of fatty acid methyl esters suitable for large clinical studies. J Lipid Res 2005;46:2299-305.

[22] Iuliano L, Pacelli A, Ciacciarelli M, et al. Plasma fatty acid lipidomics in amnestic mild cognitive impairment and Alzheimer's disease. J Alzheimers Dis 2013;36:545-53.

[23] Iuliano L, Monticolo R, Straface G, et al. Association of cholesterol oxidation and abnormalities in fatty acid metabolism in cystic fibrosis. Am J Clin Nut 2009:90:477-84.

[24] Iuliano L, Micheletta F, Natoli S, et al. Measurement of oxysterols and alphatocopherol in plasma and tissue samples as indices of oxidant stress status. Anal Biochem 2003;312:217-23.

[25] Benjamini Y, Hochberg Y. Controlling the false discovery rate: a practical and powerful approach to multiple testing. J R Stat Soc Ser B 1995;57: 289-300.

[26] Boden G. Obesity, insulin resistance and free fatty acids. Curr Opin Endocrinol Diabetes Obes 2011;18:139-43.

[27] Hodson L, Karpe F. Is there something special about palmitoleate? Curr Opin Clin Nutr Metab Care 2013;16:225-31.

[28] Vanhala M, Saltevo J, Soininen P, et al. Serum omega-6 polyunsaturated fatty acids and the metabolic syndrome: a longitudinal population-based cohort study. Am J Epidemiol 2012;176:253-60.

[29] Miyoshi N, Iuliano L, Tomono S, et al. Implications of cholesterol autoxidation products in the pathogenesis of inflammatory diseases. Biochem Biophys Res Commun 2014;446:702-8.

[30] Iuliano L. Pathways of cholesterol oxidation via non-enzymatic mechanisms. Chem Phys Lipids 2011;164:457-68.

[31] Mittendorfer B. Sexual dimorphism in human lipid metabolism. J Nut 2005;135:681-6.

[32] Alkazemi D, Egeland G, Vaya J, et al. Oxysterol as a marker of atherogenic dyslipidemia in adolescence. J Clin Endocrinol Metab 2008;93:4282-9.

[33] Wang L, Folsom AR, Zheng ZJ, et al. Plasma fatty acid composition and incidence of diabetes in middle-aged adults: the Atherosclerosis Risk in Communities (ARIC) Study. Am J Clin Nutr 2003;78:91-8.

[34] Cao H. Adipocytokines in obesity and metabolic disease. J Endocrino 2014;220:T47-59.

[35] Guillou H, Zadravec D, Martin PG, et al. The key roles of elongases and desaturases in mammalian fatty acid metabolism: Insights from transgenic mice. Prog Lipid Res 2010;49:186-99.

[36] Lee JJ, Lambert JE, Hovhannisyan Y, et al. Palmitoleic acid is elevated in fatty liver disease and reflects hepatic lipogenesis. Am J Clin Nutr 2015;101:34-43.

[37] Bodin K, Andersson U, Rystedt E, et al. Metabolism of 4 betahydroxycholesterol in humans. J Biol Chem 2002;277:31534-40.

[38] Bodin K, Bretillon L, Aden Y, et al. Antiepileptic drugs increase plasma levels of 4 beta-hydroxycholesterol in humans: evidence for involvement of cytochrome p450 3A4. J Biol Chem 2001;276:38685-9. 